\title{
目 \\ Hesitant Fuzzy MADM Approach in Optimal Selection of Investment Projects
}

\author{
Irina Khutsishvili, Gia Sirbiladze and Gvantsa Tsulaia \\ Ivane Javakhishvili Tbilisi State University, Tbilisi, Georgia \\ \{irina.khutsishvili, gia.sirbiladze, gvanca.tsulaia\}@tsu.ge
}

\begin{abstract}
The article proposes a multi-attribute decision making (MADM) approach, which is applied to the problem of optimal selection of the investment projects. This novel methodology comprises two stages. First, it makes ranking of projects based on TOPSIS (Technique for Order Preference by Similarity to Ideal Solution) method presented in hesitant fuzzy environment. We consider the case when the information on the weights of the attributes is completely unknown. The identification of the weights of the attributes is made in the context of hesitant fuzzy sets and is based on the De LucaTermini information entropy. The ranking of alternatives is made in accordance with the proximity of their distance to the positive and negative ideal solutions. Second stage of the methodology allows making the most profitable investment in several projects simultaneously. The decision on an optimal distribution of allocated investments among the selected projects is provided using the method developed by the authors for a possibilistic bicriteria optimization problem. An investment example is given to illustrate the application of the proposed approach.
\end{abstract}

\section{Introduction}

Investment decision making is based on the various special methods. Further advancement in the field led to the application of the probabilistic approach to the assessment of investment decisions [1], [2]. Along with that, many other methods were developed based on possibility analysis [3] and fuzzyset approach [4]-[10].

When there is not enough objective data, or the data is not present to make the investment decision, experienced experts (decision makers - DMs) are commissioned to solve the problem. In this case, knowledge and intellectual activities of the experts produce expert data. Thus, the analysis of investment projects involves experts' evaluations that may become dominant in decision making process.

A multi-attribute decision-making (MADM) problem deals with a selection of one alternative (decision) or several ranked alternatives involving multiple attributes. From this perspective, the investment decision-making is a MADM problem. 
Because of the inherent uncertainty of expert preferences, as well as due to the fact that objects can be fuzzy and uncertain, evaluations of attributes involved in the decision making problems most often are expressed in fuzzy numbers, triangular fuzzy numbers, confidence intervals, linguistic variables, intuitionistic fuzzy values, hesitant fuzzy elements, interval-valued hesitant fuzzy elements and so on. In this connection, many well-known MADM methods have been extended to take into account fuzzy types of attribute values [11], [12].

Nowadays there exists a large amount of literature on the theory of hesitant fuzzy sets (HFS) and their application in MADM. This paper describes novel approach, which differs from other studies and is developed based on hesitant fuzzy TOPSIS decision making model with entropy weights. The case with completely unknown weights of the attributes is examined. The attributes weights are obtained by applying De Luca-Termini non-probabilistic entropy concept [13], which is considered in the context of hesitant fuzzy sets. After that, a fuzzy hesitant TOPSIS method is applied to rank the alternatives. The developed method is applied to evaluate and rank projects in order to identify highquality projects for investment. The method is described in Section 3.

In practice, the capital is frequently invested in several projects simultaneously, each of them requiring a different credit amount. At the same time, the total investment amount is predetermined and fixed. In such cases, it becomes necessary to decide which of the projects and to what extent should share the initial investment amount. On the basis of the fuzzy hesitant TOPSIS method the projects group ranking maximum criteria is constructed. Taking into account the levels of ranking of projects group and also considering initial investment amount the possibilistic bicriteria optimization problem [14]-[17] is applied for the most advantageous investment in several projects simultaneously. From each group the projects with maximum ranking are selected, which allows for gaining maximum profit for the investment fund. The method is discussed in Section 4.

The research of the authors resulted in a new methodology and, consequently, software package development. The software package, which is based on the combined approach, was used in investment tender and supported the decision making. In Section 5 the authors provide an example clearly illustrating the work of the proposed methodology.

\section{Preliminaries}

Hesitant fuzzy set (HFS) was introduced by Torra and Narukawa in [18] and Torra in [19] as a generalization of a fuzzy set. In HFS the degree of membership of an element to a reference set is presented by several possible fuzzy values. This allows describing situations when DMs have hesitancy in providing their preferences over alternatives. The HFS is defined as follows:

Definition 1. [18,19]. Let $X$ be a finite reference set, a hesitant fuzzy set $E$ on $X$ is defined in terms of a function $h_{E}(x)$ when applied to $X$ returns a subset of $[0,1]$ :

$$
E=\left\{<x, h_{E}(x)>\mid x \in X\right\},
$$

where $h_{E}(x)$ is a set of some different values in $[0,1]$, representing the possible membership degrees of the element $x \in X$ to $E ; h_{E}(x)$ is called a hesitant fuzzy element (HFE).

Definition 2: [20]. Let $M$ and $N$ be two HFSs on $X=\left\{x_{1}, x_{2}, \ldots, x_{n}\right\}$, then the distance measure between $M$ and $N$ is defined as $d(M, N)$, which satisfies the following properties:

1). $0 \leq d(M, N) \leq 1$ 


$$
\begin{aligned}
& \text { 2). } d(M, N)=0 \text { if and only if } M=N \text {; } \\
& \text { 3). } d(M, N)=d(N, M) .
\end{aligned}
$$

It is clear that the number of values (length) for different HFEs may be different. Let $l\left(h_{E}(x)\right)$ be the length of $h_{E}(x)$. After arranging the elements of $h_{E}(x)$ in a decreasing order, let $h_{E}^{\sigma(j)}(x)$ be the jth largest value in $h_{E}(x)$. To calculate the distance between $M$ and $N$ when $l\left(h_{M}\left(x_{i}\right)\right) \neq l\left(h_{N}\left(x_{i}\right)\right)$, it is necessary extend the shorter one by adding any value in it, until both will have the same length. The choice of this value depends on the DMs' risk preferences. Optimists DMs' may add the maximum value from HFE, while pessimists may add the minimal value.

In this work the hesitant weighted Hamming distance is used that is defined by following formula

$$
d_{h w h}(M, N)=\sum_{i=1}^{n} w_{i}\left[\frac{1}{l_{x_{i}}} \sum_{j=1}^{l_{x_{i}}}\left|h_{M}^{\sigma(j)}\left(x_{i}\right)-h_{N}^{\sigma(j)}\left(x_{i}\right)\right|\right],
$$

where $h_{M}^{\sigma(j)}\left(x_{i}\right)$ and $h_{N}^{\sigma(j)}\left(x_{i}\right)$ are the $j$ th largest values in $h_{M}\left(x_{i}\right)$ and $h_{N}\left(x_{i}\right)$ respectively;

$l_{x_{i}}=\max \left\{l\left(h_{M}\left(x_{i}\right)\right), l\left(h_{N}\left(x_{i}\right)\right)\right\}$ for each $x_{i} \in X$;

$w_{i}(i=1,2, \ldots, n)$ is the weight of the element $x_{i} \in X$ such that $w_{i} \in[0,1]$ and $\sum_{i=1}^{n} w_{i}=1$.

Definition 3: [21] For a HFE $h_{E}(x)$, the score function $s\left(h_{E}(x)\right)$ is defined as follows:

$$
s\left(h_{E}(x)\right)=\sum_{j=1}^{l\left(h_{E}(x)\right)} h_{E}^{\sigma(j)}(x) / l\left(h_{E}(x)\right),
$$

where $s\left(h_{E}(x)\right) \in[0,1]$.

Let $h_{1}$ and $h_{2}$ are two HFEs. Based on score function it is possible to make ranking of HFEs according to the following rules: $h_{1}>h_{2}$, if $s\left(h_{1}\right)>s\left(h_{2}\right) ; h_{1}<h_{2}$, if $s\left(h_{1}\right)<s\left(h_{2}\right)$ and $h_{1}=h_{2}$, if $s\left(h_{1}\right)=s\left(h_{2}\right)$.

\section{Investment MADM Problem in Hesitant Fuzzy Environment}

Consider a MADM problem for investment decision making.

Assume that there are $m$ investment projects - decision making alternatives $A=\left\{A_{1}, A_{2}, \ldots, A_{m}\right\}$, and the group of DMs evaluates them with respect to an $n$ attributes $X=\left\{x_{1}, x_{2}, \ldots, x_{n}\right\}$. DMs give the evaluations over attributes in form of hesitant fuzzy numbers. Therefore, their joint assessments concerning each alternative represent HFSs.

A HFS $A_{i}$ of the $i$ th alternative on $X$ is given by

$$
A_{i}=\left\{\left\langle x_{j}, h_{A_{i}}\left(x_{j}\right)\right\rangle \mid x_{j} \in X\right\},
$$

where $h_{A_{i}}\left(x_{j}\right)=\left\{\gamma \mid \gamma \in h_{A_{i}}\left(x_{j}\right), 0 \leq \gamma \leq 1\right\}, i=1,2, \ldots, m ; j=1,2, \ldots, n$.

$h_{A_{i}}\left(x_{j}\right)$ indicates the possible membership degrees of the $i$ th alternative $A_{i}$ under the $j$ th attribute $x_{j}$, and it can be expressed as a HFE $h_{i j}$. 
Considering that the attributes have different importance degrees, the weight vector of all attributes, given by the DMs, is defined by $w=\left(w_{1}, w_{2}, \ldots, w_{n}\right)^{T}$, where $0 \leq w_{j} \leq 1, \sum_{j=1}^{n} w_{j}=1$, and $w_{j}$ is the importance degree of $j$ th attribute.

Then a hesitant MADM problem can be expressed in matrix format as follows

$$
\begin{aligned}
& \begin{array}{llll}
x_{1} & x_{2} & \cdots & x_{n}
\end{array} \\
& H=\begin{array}{c}
A_{1} \\
A_{2} \\
\vdots \\
A_{m}
\end{array}\left[\begin{array}{cccc}
h_{11} & h_{12} & \cdots & h_{1 n} \\
h_{21} & h_{22} & \cdots & h_{2 n} \\
\vdots & \vdots & \vdots & \vdots \\
h_{m 1} & h_{m 2} & \cdots & h_{m n}
\end{array}\right], w=\left(w_{1}, w_{2}, \ldots, w_{n}\right)^{T},
\end{aligned}
$$

where $H$ is the hesitant decision matrix, each element of which represents a HFE $h_{i j}$.

\subsection{Determination of the attributes weights using De Luca-Termini entropy}

Complexity and uncertainty of investment decision making problems leads to the fact that the information about attributes weights is usually incomplete or completely unknown. Here the case when the attributes weights are unknown is considered.

In this study, to identify weights of the attributes we employ objective weights method, in particular, the entropy method based on De Luca and Termini entropy concept [13] that is proposed in the context of hesitant fuzzy sets. We choose the entropy weights method based on De Luca and Termini entropy due to the fact that it is a classical entropy method for cases where expert information is processed.

De Luca and Termini [13] defined a non-probabilistic entropy formula of a fuzzy set based on Shannon's function on a finite universal set $X=\left\{x_{1}, \ldots, x_{n}\right\}$ as:

$$
E_{L T}=-k \sum_{i=1}^{n}\left[\mu_{A}\left(x_{i}\right) \ln \mu_{A}\left(x_{i}\right)+\left(1-\mu_{A}\left(x_{i}\right)\right) \ln \left(1-\mu_{A}\left(x_{i}\right)\right)\right], k>0,
$$

where $\mu_{A}: X \rightarrow[0,1] ; k$ is a positive constant.

The attributes weights definition method based on the De Luca-Termini entropy can be described as follows:

Step 1: Calculate the score matrix $S=\left(s_{i j}\right)_{m \times n}$ of hesitant decision matrix $H$, where $s_{i j}=s\left(h_{i j}\right)$ is the score value of $h_{i j}$ (see (3)).

Step2: Calculate the normalized score matrix $S^{\prime}=\left(s_{i j}^{\prime}\right)_{m \times n}$, where

$$
s_{i j}^{\prime}=s_{i j} / \sum_{i=1}^{m} s_{i j}
$$

Step3: Determine the attributes weights.

By using De Luca-Termini normalized entropy in context of hesitant fuzzy sets 


$$
\begin{gathered}
E_{j}=-\frac{1}{m \ln 2} \sum_{i=1}^{m} s_{i j}^{\prime} \ln s_{i j}^{\prime}+\left(1-s_{i j}^{\prime}\right) \ln \left(1-s_{i j}^{\prime}\right), \\
j=1,2, \ldots, n,
\end{gathered}
$$

the definition of the attributes weights is expressed by the formula

$$
w_{j}=\frac{1-E_{j}}{\sum_{j=1}^{n}\left(1-E_{j}\right)}, j=1,2, \ldots, n .
$$

where the value of $w_{j}$ represents the relative intensity of $x_{j}$ attribute importance.

\subsection{Hesitant fuzzy MADM approach based on TOPSIS method}

The idea of TOPSIS method as applied to the problem of MADM is to choose an alternative with the nearest distance from the so-called positive ideal solution (PIS) and the farthest distance from the negative ideal solution (NIS).

This Section presents a MADM approach based on the hesitant fuzzy TOPSIS with entropy weights model (proposed in Section 3 - Subsection 3.1). Different from existing extensions of TOPSIS under hesitant fuzzy environment, here the attributes of both types are considered: as attributes of benefit type, as well as attributes of cost type.

The algorithm of practical solving an investment MADM problem can be formulated as follows:

Step 1: Based on the DMs hesitant evaluations construct the aggregate hesitant decision matrix $H=\left(h_{i j}\right)_{m \times n}$.

Step2: Determine the attributes weights

$w=\left(w_{1}, w_{2}, \ldots, w_{n}\right)^{T}$ based on the method given in Section 3 (Subsection 3.1).

Step3: Determine the corresponding hesitant fuzzy PIS $A^{+}$and the hesitant fuzzy NIS $A^{-}$by formulas:

$$
\begin{aligned}
& A^{+}=\left\{\max _{i}\left\langle h_{i j}^{\sigma(\lambda)}\right\rangle\left|j \in J^{\prime} ; \min _{i}\left\langle h_{i j}^{\sigma(\lambda)}\right\rangle\right| j \in J^{\prime \prime}\right\}, \\
& A^{-}=\left\{\min _{i}\left\langle h_{i j}^{\sigma(\lambda)}\right\rangle\left|j \in J^{\prime} ; \max _{i}\left\langle h_{i j}^{\sigma(\lambda)}\right\rangle\right| j \in J^{\prime \prime}\right\},
\end{aligned}
$$

where $J^{\prime}$ is associated with a benefit attributes, and $J^{\prime \prime}$ - with a cost attributes.

Step4: Using (2) calculate the separation measures $d_{i}^{+}$and $d_{i}^{-}$of each alternative $A_{i}$ from the hesitant fuzzy PIS $A^{+}$and the hesitant fuzzy NIS $A^{-}$, respectively:

$$
\begin{aligned}
d_{i}^{+}=\sum_{j=1}^{n} d\left(h_{i j}, h_{j}^{+}\right) w_{j} & =\sum_{j=1}^{n} w_{j}\left[\frac{1}{l} \sum_{j=1}^{l}\left|h_{i j}^{\sigma(j)}-\left(h_{j}^{\sigma(j)}\right)^{+}\right|\right], \\
d_{i}^{-}=\sum_{j=1}^{n} d\left(h_{i j}, h_{j}^{-}\right) w_{j} & =\sum_{j=1}^{n} w_{j}\left[\frac{1}{l} \sum_{j=1}^{l}\left|h_{i j}^{\sigma(j)}-\left(h_{j}^{\sigma(j)}\right)^{-}\right|\right], \\
i & =1,2, \ldots, m .
\end{aligned}
$$


Step 5: Calculate the relative closeness coefficient $\delta_{i}$ of each alternative $A_{i}$ to the hesitant fuzzy PIS $A^{+}$:

$$
\delta_{i}=\frac{d_{i}^{-}}{d_{i}^{+}+d_{i}^{-}} .
$$

Step 6: Perform the ranking of the alternatives $A_{i}, i=1,2, \ldots, m$ according to the relative closeness coefficients $\delta_{i}, i=1,2, \ldots, m$ by the rule: for two alternatives $A_{\alpha}$ and $A_{\beta}$ we say $A_{\alpha} \geqslant A_{\beta}$, if $\delta_{\alpha} \geq \delta_{\beta}$, where $\geqslant$ is a preference relation on $A$.

\section{Problem of the Investment's Optimal Distribution}

Assume that after evaluation the projects with hesitant fuzzy TOPSIS method, there are $n$ ranking projects, and for each alternative (project) $A_{j}$ the ranking level $\delta_{j}$ of its choice is calculated.

We consider the issue of possible financing of the projects in $\ell$ years.

Let's assume there are additional conditions for financing the projects. In particular, it is known that

for financing of $j$ th project $j \in\{1,2, \ldots, n\}$ within $i$ th year $i \in\{1,2, \ldots, \ell\}, a_{i j}$ monetary units are required;

the profit received from implementation of $j$ th project constitutes $c_{j}$ monetary units;

$b_{i}$ monetary amount is allocated to finance projects within $i$ th year.

In practice, the amount of funding, as a rule, is insufficient to satisfy all projects. Therefore, it is supposed that for at least one $i \in\{1,2, \ldots, \ell\}$ the inequality $\sum_{j=1}^{n} a_{i j}>b_{i}$ is true.

Considering the listed constraints, we have to find an answer to the question as to which of the chosen projects should be financed to get a maximum investment profit at a minimum risk.

We offer the following solution of the problem.

If we introduce a Boolean variables $x_{j}, j \in\{1,2, \ldots, n\}$ by the rule

$$
x_{j}=\left\{\begin{array}{l}
1, \text { if the } \mathrm{j}-\text { th project is selected for finance } \\
0, \text { otherwise }
\end{array}\right.
$$

we obtain the following bicriteria Boolean linear programming problem:

$$
\left\{\begin{array}{l}
\max \sum_{j=1}^{n} \delta_{j} x_{j}, \\
\max \sum_{j=1}^{n} c_{j} x_{j}, \\
\sum_{j=1}^{n} a_{i j} x_{j} \leq b_{i}, i=1, \ldots, \ell \\
x_{j}=0 \vee 1
\end{array}\right.
$$


where the criterion (i) represents the decision on the selection of the projects' group with the maximum level of ranking; the criterion (ii) represents the decision on selection of the group of projects giving the maximum profit, while the conditions (iii) corresponds to the financial constraints.

Thus, the objective functions will be:

1) $f_{1}=\max \sum_{j=1}^{n} \delta_{j} x_{j}-$ selection the projects' group with the maximum ranking level;

2) $f_{2}=\max \sum_{j=1}^{n} c_{j} x_{j}-$ selection the projects' group ensuring a maximum profit.

To solve this problem we apply the method developed by the authors for possibilistic bicriteria optimization problems [16], [17].

In other words $X$ is the set of all Boolean vectors satisfying the conditions of the bicriteria optimization problem. Then by considering the scalar optimization problem

$$
\lambda f_{1}+(1-\lambda) f_{2} \rightarrow \max ,\left(x_{1}, x_{2}, \ldots, x_{n}\right) \in X, \lambda \in(0,1),
$$

with conditions (iii), where $\lambda$ is a weighted parameter, we can find, in the general case, some Pareto optima [14]-[17].

Thereby, the bicriteria optimization problem can be solved by linear convolution of criteria.

\section{An example of the Application of Fuzzy Decision Making Approach}

We have developed a software package supporting decision making for optimal credit granting. The decision making block consists of two main soft computing modules: the first provides the software platform for the application of the hesitant fuzzy TOPSIS method, and the second is used to solve a bicriteria optimization problem.

The software was tested on concrete data. The required information was provided by the group of 4 experts - expert commission - from the Bank of Georgia and filtered according to our demands after consultations with the managers of the Bank's crediting department.

\subsection{Comparison and Ranking the Projects Using the TOPSIS method}

Suppose that in the competition for investment five construction companies are involved. The group of DMs evaluates the investment projects taking into account the six attributes, by which the experts will score each candidate seeking an investment:

$x_{1}$ : business profitability;

$x_{2}$ : pledge guaranteeing repayment of the credit;

$x_{3}:$ location of construction object;

$x_{4}$ : workmanship;

$x_{5}$ : monthly payment of a portion of the principal and accrued interest (repayment scheme);

$x_{6}:$ percent ratio of the pledge to the credit monetary amount. 
In our concrete case all attributes are of a benefit type. DMs give evaluations in form of hesitant values. If the evaluation values of any attribute given by DMs are coincident, then such values are included in HFE only once. Assume the hesitant fuzzy decision matrix $H$ looks like Table 1:

\begin{tabular}{ccccccc}
\hline & $x_{1}$ & $x_{2}$ & $x_{3}$ & $x_{4}$ & $x_{5}$ & $x_{6}$ \\
\hline$A_{1}$ & $(0.4,0.3,0.1)$ & $(0.9,0.8,0.7,0.1)$ & $(0.9,0.6,0.5,0.3)$ & $(0.5,0.4,0.3)$ & $(0.7,0.6,0.4)$ & $(0.6,0.4,0.2)$ \\
$A_{2}$ & $(0.5,0.4)$ & $(0.9,0.7,0.6,0.3)$ & $(0.7,0.4,0.3)$ & $(0.6,0.5)$ & $(0.8,0.5,0.3)$ & $(0.7,0.5,0.3)$ \\
$A_{3}$ & $(0.3,0.2,0.1)$ & $(0.9,0.6)$ & $(0.8,0.7)$ & $(0.7,0.4,0.1)$ & $(0.9,0.7,0.5,0.4)$ & $(0.8,0.7,0.5)$ \\
$A_{4}$ & $(0.2,0.1)$ & $(0.8,0.7,0.5,0.3)$ & $(0.9,0.8,0.6)$ & $(0.8,0.5,0.4)$ & $(0.9,0.8,0.7)$ & $(0.9,0.7,0.6)$ \\
$A_{5}$ & $(0.7,0.5,0.3)$ & $(0.7,0.4,0.2)$ & $(0.9,0.7,0.6,0.4)$ & $(0.9,0.7,0.6,0.2)$ & $(0.8,0.7,0.4)$ & $(0.9,0.7,0.4)$ \\
\hline
\end{tabular}

Table 1: The Hesitant Fuzzy Decision Matrix $H$

We assume that the DMs are pessimistic, and the hesitant fuzzy data in HFEs are changed by adding the minimal values.

According to the method of determining the attributes weights given in Section 3 (Subsection 3.1), we first calculate the score matrix $S$ of hesitant decision matrix $H$ based on (3):

$$
S=\left[\begin{array}{cccccc}
0.2667 & 0.625 & 0.575 & 0.4 & 0.5667 & 0.4 \\
0.45 & 0.625 & 0.4667 & 0.55 & 0.5333 & 0.5 \\
0.2 & 0.75 & 0.75 & 0.4 & 0.625 & 0.6667 \\
0.15 & 0.575 & 0.7667 & 0.5667 & 0.8 & 0.7333 \\
0.5 & 0.4333 & 0.65 & 0.6 & 0.6333 & 0.6667
\end{array}\right]
$$

Secondly, we obtain the normalized score matrix $S^{\prime}$ using (4):

$$
S^{\prime}=\left[\begin{array}{llllll}
0.1702 & 0.2078 & 0.1792 & 0.1589 & 0.1794 & 0.1348 \\
0.2872 & 0.2078 & 0.1455 & 0.2185 & 0.1689 & 0.1685 \\
0.1277 & 0.2493 & 0.2338 & 0.1589 & 0.1979 & 0.2247 \\
0.0957 & 0.1911 & 0.2390 & 0.2252 & 0.2533 & 0.2472 \\
0.3191 & 0.1440 & 0.2026 & 0.2384 & 0.2005 & 0.2247
\end{array}\right]
$$

Then the weighting vector of attributes is determined using (5) and (6):

$$
w=(0.180951,0.163632,0.163799,0.163653,0.162714,0.165251)^{T}
$$

Following the hesitant fuzzy TOPSIS method, we determine the hesitant fuzzy PIS $A^{+}$and the hesitant fuzzy NIS $A^{-}$by (7) and (8), respectively:

$$
\begin{gathered}
A^{+}=\{(0.7,0.5,0.4,0.4),(0.9,0.8,0.7,0.6),(0.9,0.8,0.7,0.7), \\
(0.9,0.7,0.6,0.5),(0.9,0.8,0.7,0.7),(0.9,0.7,0.6,0.6)\}, \\
A^{-}=\{(0.2,0.1,0.1,0.1),(0.7,0.4,0.2,0.1),(0.7,0.4,0.3,0.3), \\
(0.5,0.4,0.1,0.1),(0.7,0.5,0.3,0.3),(0.6,0.4,0.2,0.2)\} .
\end{gathered}
$$

Here, we take into account that all attributes are of a benefit type. 
Then we calculate the distances $d_{i}^{+}$and $d_{i}^{-}$of each alternative $A_{i}$ from the hesitant fuzzy PIS $A^{+}$and the hesitant fuzzy NIS $A^{-}$by formulas (9) and (10), respectively:

$$
\begin{aligned}
& d_{1}^{+}=0.250588, \quad d_{2}^{+}=0.20603, \quad d_{3}^{+}=0.173351, \quad d_{4}^{+}=0.12923, \quad d_{5}^{+}=0.152226 ; \\
& d_{1}^{-}=0.116233, \quad d_{2}^{-}=0.16079, \quad d_{3}^{-}=0.193469, \quad d_{4}^{-}=0.23759, \quad d_{5}^{-}=0.214594 \text {. }
\end{aligned}
$$

Using (11) to calculate the relative closeness coefficient $\delta_{i}$ of each alternative $A_{i}$ to the hesitant fuzzy PIS $A^{+}$we obtain:

$$
\delta_{1}=0.316865, \quad \delta_{2}=0.438335, \quad \delta_{3}=0.527423, \quad \delta_{4}=0.647702, \quad \delta_{5}=0.585011 .
$$

Finally, we perform the ranking of the alternatives $A_{i}, i=1,2, \ldots, 5$ according to the relative closeness coefficients $\delta_{i}$ and obtain:

$$
A_{4} \succ A_{5} \succ A_{3} \succ A_{2} \succ A_{1} .
$$

This means that when investing the capital only in one project, DMs prefer to the investment project $A_{4}$, i.e. the project $A_{4}$ receive investment.

Frequently, the investment amount has to be distributed among several projects. We can do this in the second stage of the proposed approach.

\subsection{Problem of the Optimal Distribution of Investment}

Using the formulas (12)-(13) we will deal with the possibilistic bicriteria optimization problem allowing for the most profitable investments into a number of projects.

Bank considers an investment that totals to $\$ 150$ million over four years $(i=1,2,3,4), \$ 40$ million in first three years $\left(b_{i}=40, i=1,2,3\right)$ and $\$ 30$ million in fourth year.

The values $a_{i j}$ of investments, that are required for $j$ th project in $i$ th year, as well as the $c_{j}$ magnitudes of profits from the realization of $j$ th project during four years are shown in the following table (see Table 2):

\begin{tabular}{ccccccc}
\hline \multicolumn{3}{c}{ Years } & \multicolumn{5}{c}{ Projects } \\
& & $A_{1}$ & $A_{2}$ & $A_{3}$ & $A_{4}$ & $A_{5}$ \\
\hline \multirow{3}{*}{$a_{i j}$} & 1 & 10 & 10 & 12 & 18 & 8 \\
& 2 & 6 & 14 & 16 & 20 & 12 \\
& 3 & 10 & 10 & 14 & 20 & 10 \\
& 4 & 10 & 9 & 10 & 10 & 5 \\
\hline$c_{j}$ & & 25 & 20 & 30 & 40 & 18 \\
\hline
\end{tabular}

Table 2: The values of $a_{i j}$ and $c_{j}$

We use obtained distribution of projects ranking levels (14), the information given in Table 2, and solve problem (12)-(13). The objective functions guaranteeing, respectively, maximum ranking levels of the selected projects and a maximum profit are as follows:

$$
f_{1}=0.316865 \cdot x_{1}+0.438335 \cdot x_{2}+0.527423 \cdot x_{3}+0.647702 \cdot x_{4}+0.585011 \cdot x_{5} ;
$$




$$
f_{2}=25 \cdot x_{1}+20 \cdot x_{2}+30 \cdot x_{3}+40 \cdot x_{4}+18 \cdot x_{5} .
$$

Using the relation $\bar{f}_{i}=f_{i} / f_{i}^{*}$, where $f_{i}^{*}=\max f_{i}, i=1,2$, in order to pass over to dimensionless values, we obtain

$$
\begin{aligned}
& \bar{f}_{1}=0.204484 \cdot x_{1}+0.282873 \cdot x_{2}+0.340365 \cdot x_{3}+0.417986 \cdot x_{4}+0.377529 \cdot x_{5} \\
& \bar{f}_{2}=0.294118 \cdot x_{1}+0.235294 \cdot x_{2}+0.352941 \cdot x_{3}+0.470588 \cdot x_{4}+0.211765 \cdot x_{5} .
\end{aligned}
$$

By solving problem (12)-(13) for $\bar{f}_{1}$ and $\bar{f}_{2}$ we can see that, in general, its solution depends on a choice of the parameter $\lambda$ (see (13)). In particular, for $\lambda \in[0 ; 0.1]$ we obtain the set of Boolean variables $\{1,1,0,1,0\}$, and an optimal solution is the selection of projects' group $\left(A_{1}, A_{2}, A_{4}\right)$, and for $\lambda \in[0.2 ; 1]$ the set of Boolean variables is $\{1,0,0,1,1\}$, and an optimal solution is the selection of projects' group ( $\left.A_{1}, A_{4}, A_{5}\right)$.

The choice of $\lambda$ from the interval $[0 ; 0.1]$ means that the DMs are more inclined to the objective criterion $f_{2}$, whereas other chosen values of $\lambda$ mean that the DMs are inclined to the subjective criterion $f_{1}$.

If the group of projects $\left(A_{1}, A_{2}, A_{4}\right)$ is given a credit and investment over the years is $\$ 38$ million in the first year, $\$ 40$ million in the second and the third years, and $\$ 29$ million in the fourth year, the bank will gain $\$ 85$ million profit in four years.

If the group of projects $\left(A_{1}, A_{4}, A_{5}\right)$ is given a credit and investment over the years is distributed as $-\$ 36$ million in the first year, $\$ 38$ million in the second year, $\$ 40$ million in the third year, and $\$$ 25 million in the fourth year - in four years a total profit of the bank will be $\$ 83$ million.

\section{Conclusion}

In this paper the novel approach for solving MADM problem based on hesitant fuzzy TOPSIS method with entropy weights is developed.

In our methodology have been used new aspects. We proposed a novel attribute weighting method in context of hesitant fuzzy sets. It is based on De Luca and Termini information entropy to express the relative intensities of attribute importance and to determine the weights of attributes. There are many methods of the applicability of the TOPSIS approach under hesitant environment. In our work we proposed the formulas to calculate PIS and NIS, which take into account as the attributes of a benefits type, as well as the attributes of a cost type.

The developed approach was applied in the problem of investment decision making with the aim of optimal distribution of investment among several of projects. The decision on an optimal distribution of investments is provided on the basis of the method developed by authors for possibilistic bicriteria optimization problems.

Based on proposed two-stage methodology we have developed software package, which is used in real investment decision making problem. The application and testing of the software was carried out based on the data provided by the "Bank of Georgia". The results are illustrated in the example. 


\section{References}

[1] Merigó, J.M. (2009). Probabilistic Decision Making with the OWA Operator and its Application in Investment Management. In Proceedings of the Conference European Society for Fuzzy Logic and Technology - EUSFLAT, Lisbon, Portugal, 1364-1369.

[2] Merigó, J.M. (2011). Fuzzy Multi-Person Decision Making with Fuzzy Probabilistic Aggregation Operators. Int. Journal of Fuzzy Systems, 13, pp. 163-174.

[3] Dubois, D. and Prade, H. (1988). Possibility Theory: An Approach to Computerized Processing of Uncertainty. New-York: Plenum Press.

[4] Bellman, R.E. and Zadeh, L.A. (1970). Decision-Making in a Fuzzy Environment. Management Science, 17(4), B-141-B-164.

[5] Ruan, D., Kacprzyk, J. and Fedrizzi M. (2001). Soft Computing for Risk Evaluation and Management: Applications in Technology, Environmenf and Finance. Studies in Fuzziness and Soft Computing. Heidelberg, New York: Physica-Verlag.

[6] Yager, R.R. (1988). On Ordered Weighted Averaging aggregation operators in multicriteria decision making. IEEE Trans. On Systems, Man and Cybernetics B, 18, pp. 183-190.

[7] Yager, R.R., Kacprzyk, J., and Beliakov, G. (2011). Recent Development in the ordered Weighted Averaging Operations: Theory and Practice. Studies in Fuzziness and Soft Computing 265. Berlin: Springer-Verlag.

[8] Khutsishvili, I. and Sirbiladze, G. (2009). Decision Support's Precising Technology in the Investment Project Risk Management. In Proceedings of the 11th WSEAS International Conference on Automatic Control, Modelling and Simulation (ACMOS '09), Istanbul, Turkey, pp. 303-311.

[9] Sirbiladze, G., Khutsisvili, I., and Dvalishvili, P. (2010). Decision Precising Fuzzy Technology to Evaluate the Credit Risks of Investment Projects. In Proceedings of IEEE 10-th International Conference on Intelligent Systems Desigh and Applications (ISDA 2010), Cairo, Egypt, pp. 103108.

[10] Sirbiladze, G., Khutsishvili, I., and Ghvaberidze, B. (2014). Multistage decision-making fuzzy methodology for optimal investments based on experts' evaluations. European Journal of Operational Research, vol. 232, issue 1, pp. 169-177.

[11] Xu, Z., and Zhang, X. (2013). Hesitant fuzzy multi-attribute decision making based on TOPSIS with incomplete weight information. Knowledge-Based Systems 52, pp. 53-64.

[12] Tian, J., Yu, D., Yu, B., and Ma, S. (2013). A fuzzy TOPSIS model via chi-square test for information source selection. Knowledge-Based Systems 37, pp. 515-527.

[13] De Luca, A., and Termini, S. (1972). A Definition of a Nonprobabilistic Entropy in the Setting of Fuzzy Sets Theory. Information and Control 20, pp. 301-312.

[14] Ehrgott, M. (2005). Multicriteria optimization. 2nd Edition. Berlin Heidelberg: Springer.

[15] Taha, A. Hamdy. (2011). Operations Research: An Introduction. 9th Edition. Upper Saddle River, N.J.: Pearson/Prentice Hall.

[16] Sirbiladze, G., Ghvaberidze, B., Latsabidze, T., and Matsaberidze, B. (2009). Using a minimal fuzzy covering in decision-making problems. Information Sciences, 179 (12), pp. 2022-2027.

[17] Sirbiladze, G., Sikharulidze, A., Ghvaberidze, B., and Matsaberidze, B. (2011). Fuzzyprobabilistic Aggregations in the Discrete Covering Problem. International Journal of General Systems, 40(2), pp. 169 -196.

[18] Torra, V., and Narukawa, Y. (2009). On hesitant fuzzy sets and decision. In: The 18th IEEE International Conference on Fuzzy Systems, Jeju Island, Korea, pp. 1378-1382.

[19] Torra, V. (2010). Hesitant fuzzy sets. International Journal of Intelligent Systems 25, pp. 529539. 
[20] Xu, Z., and Xia, M. (2011). Distance and similarity measures for hesitant fuzzy sets. Information Sciences 181, pp. 2128-2138.

[21] Xia, M., and Xu, Z. (2011) Hesitant fuzzy information aggregation in decision making. International Journal of Approximate Reasoning, vol. 52, no. 3, pp. 395-407. 